\title{
FACTORES QUE INFLUYEN EN LA ABUNDANCIA DE LARVAS DE COLEOPTERA: MELOLONTHIDAE CON IMPORTANCIA AGRÍCOLA EN LA REGIÓN PURHÉPECHA, MICHOACÁN, MÉXICO
}

\author{
Esperanza PÉREZ-Agis, ${ }^{1}$ Miguel Ángel MORÓn, ${ }^{2 *}$ Miguel B. NÁJERA- \\ RINCÓN ${ }^{3}$ \& ADRIANA E. CASTRO-RAMÍREZ ${ }^{4}$ \\ ${ }^{1}$ Programa de Aprovechamiento Integral de Recursos Naturales Occidente A.C. <pairmich@gmail. \\ com> \\ ${ }^{2}$ Red de Biodiversidad y Sistemática, Instituto de Ecología A.C. Apdo. Postal 63, Xalapa, Veracruz \\ 91000 México. \\ ${ }^{3}$ Campo Experimental Uruapan, CIRPAC-INIFAP, Uruapan, Michoacán 60500 México. \\ ${ }^{4}$ Departamento de Agroecología, ECOSUR, Apdo. Postal 63, San Cristóbal de las Casas, Chiapas \\ 29290 México. \\ *Autor de correspondencia: <miguel.moron@inecol.edu.mx >
}

Pérez-Agis, E., Morón, M. A., Nájera-Rincón, M. B. \& Castro-Ramírez, A. E. 2014. Factores que influyen en la abundancia de larvas de Coleoptera: Melolonthidae con importancia agrícola en la región Purhépecha, Michoacán, México. Acta Zoológica Mexicana (n.s.), 30(1): 161-173.

RESUMEN. Para definir el nivel de influencia de los factores ambientales y del manejo del agroecosistema en el complejo de larvas de Coleoptera Melolonthidae en dos localidades productoras de maíz en la Región Purhépecha de Michoacán, México, una con manejo extensivo (San Felipe) y la otra con manejo intensivo (Uricho), se seleccionaron cuatro tipos de factores que pueden influir en su distribución: i) físicos, ii) manejo de la fertilidad del suelo, iii) biológicos, y iv) temperatura y precipitación del área. Se integró una matriz de datos formada por 25 variables en ocho sitios de muestreo. Para identificar alguna tendencia de agrupación de las especies, se aplicó un análisis de correspondencia sin tendencia. Los resultados muestran que la agrupación de las especies en el sistema con manejo extensivo (San Felipe) se explica en un $62 \%$ de probabilidad por el periodo de descanso con libre pastoreo y la temperatura mínima de invierno. Mientras que en el sistema de manejo intensivo (Uricho) la agrupación de las especies se explica con un $62 \%$ de probabilidad por la aplicación de herbicidas. Otras variables relacionadas son los porcentajes de materia orgánica y de humedad de 0 a 15 centímetros de profundidad, especialmente en una de las localidades con un $68.4 \%$ de probabilidad.

Palabras clave: análisis de correspondencia sin tendencia, gallina ciega, sistemas de manejo en maíz.

Pérez-Agis, E., Morón, M. A., Nájera-Rincón, M. B. \& Castro-Ramírez, A. E. 2014. Influence of management factors on the abundance of larvae of Coleoptera: Melolonthidae with agricultural

Recibido 18/06/2013; aceptado: 08/10/2013. 
importance at Purhepecha Region, Michoacan, Mexico. Acta Zoológica Mexicana (n.s.), 30(1): 161-173.

ABSTRACT. The influence of the environmental and management factors on the white grub complex in two localities at Purhepecha Region in the state of Michoacán, Mexico was studied. Maize fields at San Felipe are under extensive management, and maize fields at Uricho are under intensive management. Four factors that could influence on the distribution of the species of Coleoptera Melolonthidae were selected: i) soil, humidity and organic matter percentage, ii) fertility management, iii) biological, abundance of 10 species, iv) temperature and precipitation in the area. One matrix with information of 25 variables and eight sites was formed for each one of two localities. The information was analyzed by Detrended Correspondence Analysis. As part of the results we found that the variables that explain the grouping of the species of white grubs at the extensive system (San Felipe) are repose with free pasturing and minimum winter temperature with $62 \%$ of probability. While intensive system (Uricho) species grouping explained with $62 \%$ probability by the application of herbicides. The second variables in importance are organic matter and soil humidity percentage at 15 centimeter depth with $68.41 \%$ of probability.

Key words: Detrended correspondence analysis, white grubs, management systems in maize.

\section{INTRODUCCIÓN}

Se sabe que los estados larvarios de algunos escarabajos de la familia Melolonthidae son una de las principales plagas rizófagas de las gramíneas, especialmente de maíz en la República Mexicana y Latinoamérica. Dichas especies dañinas forman parte de un conjunto de especies al que se le ha llamado "complejo gallina ciega" (CGC), que también incluye especies benéficas (Morón 1997; Ramírez \& Castro 2000; Romero et al. 2010). Sólo una minoría de las especies que conforman el complejo se ha comprobado que son rizófagas y que por su capacidad de adaptación a ambientes alterados se consideran como plaga de los cultivos. Cabe mencionar que aún hay mucha discusión sobre el grado de especificidad de los hábitos de muchas de estas especies o su capacidad de hacer de este hábito algo más facultativo de acuerdo a la oferta alimenticia y condiciones de vida (Morón 2010).

Según Büchs (2003) los fitófagos son sensibles, en orden decreciente, a: intensidad de manejo, estructura del paisaje, conectividad del hábitat, ocurrencia y abundancia de herbívoros mono y polífagos. Sin embargo, evaluar la influencia de estos factores en las especies edafícolas plantea un notable reto en la práctica. Pero abordar el tema adquiere mucha importancia porque recientemente se ha considerado al suelo como un sistema clave en el funcionamiento de los ecosistemas terrestres, debido a que en él se llevan a cabo procesos vitales controlados por la actividad biológica, la cual depende de la temperatura y la humedad (Fragoso et al. 2001).

Como un complemento a los resultados de la investigación desarrollada por Pérez-Agis y colaboradores (2008), el propósito del presente estudio consiste en evaluar la influencia de algunos factores ambientales y del manejo del agroecosistema en el complejo de larvas de Coleoptera Melolonthidae en localidades productoras de maíz con manejo extensivo o intensivo en la región Purhépecha de Michoacán, México. 
Debido a las particularidades del suelo se le ha considerado como un sistema en sí mismo, una de las razones señalada por Büchs (2003) es que los procesos en el suelo son más lentos en comparación con otros componentes del agroecosistema, así que las prácticas de manejo que alteran el medio físico son las que más pueden influir en las especies que habitan este medio (Brown et al. 2001).

\section{MATERIALES Y MÉTODOS}

El estudio se llevó a cabo en dos localidades de la Región Purhépecha del estado de Michoacán: San Francisco Uricho, Municipio de Erongarícuaro y San Felipe de los Herreros, Municipio de Charapan. En cada localidad se seleccionaron cuatro parcelas como sitio de muestreo permanente durante 10 meses (enero a octubre del 2004), donde se muestreó mensualmente la presencia de larvas de gallina ciega y suelo a dos profundidades. Ambos sitios se seleccionaron por producir maíz bajo dos condiciones contrastantes de manejo, en San Felipe de los Herreros el manejo es extensivo que consiste en labranza convencional con tracción mixta (mecánica + animal), periodos de descanso de 1 a 2 años entre cada ciclo de cultivo con libre pastoreo, fertilización a base de enmiendas orgánicas, mano de obra familiar y sin uso de plaguicidas para el control de plagas y malezas. Y en San Francisco Uricho el manejo es intensivo que consiste en labranza reducida a mínima con tracción mecánica, sin periodos de descanso, pero con rotación de cultivos (Zea mays - Vicia sativa) entre cada ciclo de cultivo, empleo de mano de obra familiar, fertilización química y aplicación de herbicidas para el control de malezas (Cuadro 1).

Las variables a analizar fueron: porcentaje de humedad del suelo en los estratos de 0 a 15 centímetros y de 15 a 30 centímetros de profundidad, el cual se calculó mensualmente (enero a octubre 2004) por diferencia de peso de muestras frescas y secas, después del secado de las muestras por 16 horas a $90^{\circ} \mathrm{C}$ en las instalaciones del laboratorio de microbiología de suelos de la Universidad Michoacana de San Nicolás de Hidalgo (UMSNH). Porcentaje de materia orgánica en los estratos 0 a 15 y de 15 a 30 centímetros de profundidad, el cual se determinó en las muestras de suelo del vía húmeda por digestión de cromáto de potasio y ácido sulfúrico con el método de Walkley \& Black que se realizo en al año 2006 en el laboratorio de suelos del INIFAP-Morelia. Como variables ambientales se seleccionaron: temperaturas de verano y de invierno máxima y mínima; precipitación media de verano y de invierno máxima y mínima, de las estaciones meteorológicas de Patamban $\left(19^{\circ} 53^{\prime} \mathrm{N}, 102^{\circ} 13^{\prime} \mathrm{O}\right.$, $1700 \mathrm{msnm}$ ) durante el período 1981-2006, y Pátzcuaro (19³ 3' $\mathrm{N}, 101^{\circ} 36^{\prime} \mathrm{O}, 2035$ msnm) en el periodo1991-2006 a cargo de la SAGARPA. Como variable de manejo del suelo se seleccionaron: aplicación de estiércol o fertilizante químico, periodo de descanso con libre pastoreo y aplicación de herbicida e insecticidas. Y finalmente como variables biológicas se consideró la abundancia registrada de las 10 especies de Melolonthidae encontradas en ambas localidades (Pérez-Agis et al. 2008), cinco 
Pérez-Agis et al.: Abundancia de larvas de Melolonthidae en Michoacán, México

Cuadro 1. Datos de las parcelas de muestreo en dos localidades de la Región Purhépecha, Michoacán (2004).

\begin{tabular}{|c|c|c|c|c|c|}
\hline \multicolumn{6}{|c|}{ Parcelas del Ejido San Francisco Uricho, Erongaricuaro } \\
\hline $\begin{array}{c}\text { Clave } \\
\text { de } \\
\text { caso }\end{array}$ & Productor & Paraje & $\begin{array}{l}\text { Tipo de } \\
\text { suelo }\end{array}$ & Relieve & Patrón de cultivos* \\
\hline u1 & Rolando Pérez & Porumbo & Andosol & Plano & avena - maíz \\
\hline $\mathrm{u} 2$ & Alberto Jorge & Porumbo & Andosol & Plano & Trigo + Vicia sativa -maíz \\
\hline u3 & Mauro & Porumbo & Andosol & Plano & trigo-maíz híbrido \\
\hline $\mathrm{u} 4$ & Valentín & Porumbo & Andosol & Plano & Vicia sativa -maíz \\
\hline \multicolumn{6}{|c|}{ Parcelas de la Comunidad Indígena San Felipe de los Herreros, Charapan } \\
\hline $\begin{array}{c}\text { Clave } \\
\text { de } \\
\text { caso }\end{array}$ & Productor & Paraje & $\begin{array}{l}\text { Tipo de } \\
\text { suelo }\end{array}$ & Relieve & Patrón de cultivos \\
\hline sf1 & Simon Alonso & $\begin{array}{l}\text { Ladera } \\
\text { Huariticuaro }\end{array}$ & Andosol & Plano & maíz-descanso \\
\hline $\mathrm{sf2}$ & $\begin{array}{l}\text { Donaciano Arévalo } \\
\text { Fuentes }\end{array}$ & $\begin{array}{l}\text { Plan de } \\
\text { Huariticuaro }\end{array}$ & Andosol & Plano & maíz-descanso \\
\hline sf3 & Daniel Campos & $\begin{array}{l}\text { Ladera } \\
\text { Perendícuaro }\end{array}$ & Andosol & ladera fuerte & avena-maíz \\
\hline sf4 & Jorge Vargas & $\begin{array}{l}\text { Ladera } \\
\text { Perendícuaro }\end{array}$ & Andosol & ladera fuerte & maíz-descanso \\
\hline
\end{tabular}

*Patrón de cultivos: (-) significa la secuencia de cultivos en rotación o tiempo sin cultivar (descanso), los ciclos en los cultivos son primavera - verano y otoño - invierno del mismo año, mientras que en el descanso el terreno se emplea para libre pastoreo y puede durar al menos un año. (+) Significa asociación en el mismo lugar y mismo periodo.

de ellas solo se identificaron a nivel de morfoespecie: Macrodactylus sp. (sp. 2), Phyllophaga sp. P4 (sp. 4), Paranomala sp. A2 (sp. 6), Paranomala sp. A1 (sp. 10) y Diplotaxis sp. (sp. 9), y cinco a nivel de especie: Isonychus arizonensis Howden (sp. 1), Phyllophaga dentex (Bates) (sp. 3), Phyllophaga platyrhina (Bates) (sp. 5), Paranomala punctatipennis (Blanchard) (sp. 7), y Phyllophaga vetula (Horn) (sp. 8).

Para el análisis de las variables seleccionadas (Cuadro 2) se empleó el análisis multivariado de Correspondencia sin Tendencia (Detrended Correspondence Analysis, DCA). Este es un análisis de gradientes indirecto, que permite relacionar la composición de las especies y sitios con características ambientales definidas o indirectas. Este análisis permite también identificar patrones de distribución en un gradiente de indicadores ambientales. Comparado con un análisis de regresión el DCA es menos detallado, pero brinda información confiable con suficiente detalle para definir patrones de distribución, especialmente cuando se conocen las variables que determinan la 
Cuadro 2. Valor de las variables latentes para Análisis de Correspondencia sin tendencia para MVSP 3.0 Complejo Gallina Ciega en dos localidades de la Región Purhépecha de Michoacán, 2007.

\begin{tabular}{|c|c|c|c|c|c|c|c|}
\hline parcela & $\begin{array}{c}\text { Humedad } \\
0-15 \mathrm{~cm} \\
(\%)\end{array}$ & $\begin{array}{c}\text { Humedad } \\
15-30 \mathrm{~cm} \\
(\%)\end{array}$ & $\begin{array}{c}\text { Materia } \\
\text { Orgánica } \\
0-15 \mathrm{~cm} \\
(\%)\end{array}$ & $\begin{array}{c}\text { Materia } \\
\text { Orgánica } \\
15-30 \mathrm{~cm} \\
(\%)\end{array}$ & $\begin{array}{l}\text { Temp. } \\
\text { verano } \\
\text { máxima } \\
\left({ }^{\circ} \mathrm{C}\right)\end{array}$ & $\begin{array}{l}\text { Temp. } \\
\text { verano } \\
\text { mínima } \\
\left({ }^{\circ} \mathrm{C}\right)\end{array}$ & $\begin{array}{l}\text { Temp. } \\
\text { invierno } \\
\text { máxima } \\
\left({ }^{\circ} \mathrm{C}\right)\end{array}$ \\
\hline u1 & 13.31 & 13.77 & 5.35 & 5.24 & 34.26 & 7.91 & 28.8 \\
\hline $\mathbf{u} 2$ & 13.83 & 14.34 & 4.57 & 4.57 & 34.26 & 7.91 & 28.8 \\
\hline u3 & 12.64 & 13.08 & 4.01 & 4.46 & 34.26 & 7.91 & 28.8 \\
\hline u4 & 13.17 & 13.91 & 4.01 & 3.9 & 34.26 & 7.91 & 28.8 \\
\hline sf1 & 15.28 & 14.99 & 2.9 & 3.35 & 24.93 & 7.99 & 24.32 \\
\hline sf2 & 12.87 & 13.32 & 3.35 & 2.68 & 24.93 & 7.99 & 24.32 \\
\hline sf3 & 15.32 & 16.43 & 2.01 & 3.35 & 24.93 & 7.99 & 24.32 \\
\hline sf4 & 15.9 & 16.62 & 2.9 & 2.79 & 24.93 & 7.99 & 24.32 \\
\hline \multicolumn{8}{|c|}{ continua ... } \\
\hline parcela & $\begin{array}{c}\text { Temp. inv } \\
\text { minima } \\
\left({ }^{\circ} \mathrm{C}\right)\end{array}$ & $\begin{array}{l}\text { Pp media } \\
\text { verano } \\
(\mathrm{mm})\end{array}$ & $\begin{array}{c}\text { Pp media } \\
\text { invierno } \\
(\mathrm{mm})\end{array}$ & $\begin{array}{c}\text { Fertilizante } \\
\text { orgánico } \\
\text { (presencia } \\
\text { /ausencia) }\end{array}$ & $\begin{array}{l}\text { Fertilizante } \\
\text { químico } \\
\text { (presencia } \\
\text { /ausencia) }\end{array}$ & $\begin{array}{l}\text { Herbicida } \\
\text { (presencia } \\
\text { /ausencia) }\end{array}$ & $\begin{array}{l}\text { Insecticida } \\
\text { (presencia } \\
\text { /ausencia) }\end{array}$ \\
\hline u1 & 0.47 & 174.17 & 15.22 & 0 & 1 & 1 & 1 \\
\hline $\mathbf{u} 2$ & 0.47 & 174.17 & 15.22 & 0 & 1 & 1 & 0 \\
\hline $\mathbf{u} 3$ & 0.47 & 174.17 & 15.22 & 0 & 1 & 1 & 1 \\
\hline u4 & 0.47 & 174.17 & 15.22 & 1 & 0 & 1 & 0 \\
\hline sf1 & 1.19 & 226.73 & 14.78 & 0 & 1 & 0 & 0 \\
\hline sf 2 & 1.19 & 226.73 & 14.78 & 1 & 0 & 0 & 0 \\
\hline $\mathbf{s f 3}$ & 1.19 & 226.73 & 14.78 & 0 & 1 & 0 & 0 \\
\hline sf4 & 1.19 & 226.73 & 14.78 & 1 & 0 & 0 & 0 \\
\hline \multicolumn{8}{|c|}{ continua ... } \\
\hline parcela & $\begin{array}{l}\text { Descanso } \\
\text { (presencia } \\
\text { /ausencia) }\end{array}$ & $\mathrm{sp} 1$ & $\mathrm{sp} 2$ & sp3 & $\mathrm{sp} 4$ & $\mathrm{sp} 5$ & sp6 \\
\hline u1 & 0 & 2 & 10 & 1 & 0 & 0 & 0 \\
\hline $\mathbf{u} 2$ & 0 & 0 & 31 & 1 & 4 & 1 & 0 \\
\hline $\mathbf{u} 3$ & 0 & 0 & 0 & 3 & 0 & 0 & 0 \\
\hline u4 & 0 & 1 & 45 & 17 & 4 & 0 & 3 \\
\hline sf1 & 1 & 0 & 8 & 0 & 0 & 10 & 0 \\
\hline sf2 & 1 & 0 & 5 & 0 & 0 & 12 & 0 \\
\hline sf3 & 1 & 0 & 4 & 0 & 0 & 2 & 0 \\
\hline sf4 & 1 & 0 & 2 & 0 & 0 & 19 & 0 \\
\hline
\end{tabular}


Cuadro 2. Continúa.

\begin{tabular}{ccccc} 
& & \multicolumn{3}{r}{ continua $\ldots$} \\
parcela & $\mathrm{sp} 7$ & $\mathrm{sp} 8$ & $\mathrm{sp} 9$ & $\mathrm{sp} 10$ \\
$\mathbf{u 1}$ & 0 & 0 & 0 & 0 \\
$\mathbf{u 2}$ & 0 & 0 & 0 & 0 \\
$\mathbf{u 3}$ & 0 & 0 & 0 & 0 \\
$\mathbf{u 4}$ & 0 & 0 & 0 & 0 \\
$\mathbf{f f 1}$ & 2 & 1 & 2 & 0 \\
$\mathbf{s f 2}$ & 1 & 3 & 1 & 5 \\
$\mathbf{s f 3}$ & 1 & 0 & 8 & 2 \\
$\mathbf{s f 4}$ & 4 & 1 & 0 & 1 \\
\hline
\end{tabular}

ocurrencia de las especies. El cálculo se realizó con el programa Multivariate Statical Package versión 3.0.

El resultado del DCA es un ejercicio de ordenación en una gráfica bidimensional, en donde los sitios están representados por puntos, de manera que los puntos que están cercanos corresponden a sitios con una composición similar de especies, y los puntos que están alejados corresponden a sitios con una composición de especies poco semejante (Braak 1995). Para el análisis se construye una variable teórica que explica mejor la ocurrencia de los datos de las especies, mediante la selección del valor promedio (weighted averagin algorithm) de los sitios que mejor explica la dispersión de la puntuación de las especies. La dispersión de la puntuación se estabiliza después de 10 ciclos de iteración, al mismo tiempo, tanto las especies como los sitios se estabilizan. El resultado es un valor que expresa la máxima dispersión en cada eje. El primer eje contiene la puntuación más alta y así va disminuyendo sucesivamente, lo deseable es que en los primeros tres ejes se acumule $70 \%$ de la dispersión de los datos. En matemáticas los ejes de ordenación son llamados eigenvectores, que es un conjunto de valores que denotan un espacio multidimensional en sí mismo (eigen en alemán $=$ el mismo). Cada eigenvector tiene un eigenvalor denominado $\lambda$ (lamda) que representa la máxima puntuación de dispersión de las especies en los ejes de ordenación (idem).

\section{RESULTADOS}

Depuración de variables.- Inicialmente se corrió el programa con las 25 variables seleccionadas (Cuadro 2), aunque se mostró una tendencia de agrupación, apenas se acumuló el $65.13 \%$ de probabilidad en los tres primeros eigemvectores, por lo que se procedió a la depuración de las variables con menor puntuación, éstas fueron: porcentaje de materia orgánica de 15 a $30 \mathrm{~cm}$, temperatura de verano máxima y mínima, 
precipitación media de invierno, fertilizante orgánico e insecticida. Esto permitió aumentar la confiabilidad de los resultados en los primeros tres ejes de la Matriz 1, en la cual se registró un aumento de tres puntos porcentuales, de manera que se acumuló el $69.4 \%$ en los primeros tres ejes.

Variables que influyen en la composición del CGC.- Con un $62 \%$ de probabilidad acumulada en el primer eje, las variables que explican mejor la agrupación de larvas de Melolonthidae son: libre pastoreo $(\lambda 2.60)$ y temperatura de invierno $(\lambda 1.26)$ para las especies presentes en el sistema de manejo extensivo y aplicación de herbicida $(\lambda-0.75)$ en el de manejo intensivo. Las variables que explicaron la agrupación, con un $69.4 \%$ de probabilidad acumulada en el tercer eje son: porcentaje de humedad del suelo de 0 a $15 \mathrm{~cm}$ de profundidad $(\lambda 0.40)$ y porcentaje de materia orgánica en el suelo de 0 a $15 \mathrm{~cm}(\lambda 0.64)$.

La especies del CGC forman tres agrupaciones (Fig. 1), la primera hacia la derecha en el cuadrante positivo, donde el pastoreo y la temperatura de invierno mínima promedio parecen explicar el agrupamiento de sp. 8 (P. vetula), sp. 7 (Pa. punctatipennis) y sp. 5 ( $P$. platyrhina) especies presentes en San Felipe de los Herreros, localidad con manejo extensivo para la producción de maíz. Del mismo lado, en el cuadrante negativo, los porcentajes de materia orgánica de 0 a $15 \mathrm{~cm}$ de profundidad del suelo y de humedad a la misma profundidad parecen explicar la agrupación de sp. 9 (Diplotaxis sp.) y sp. 10 (Paranomala sp. 1) ambas especies sólo registradas en San

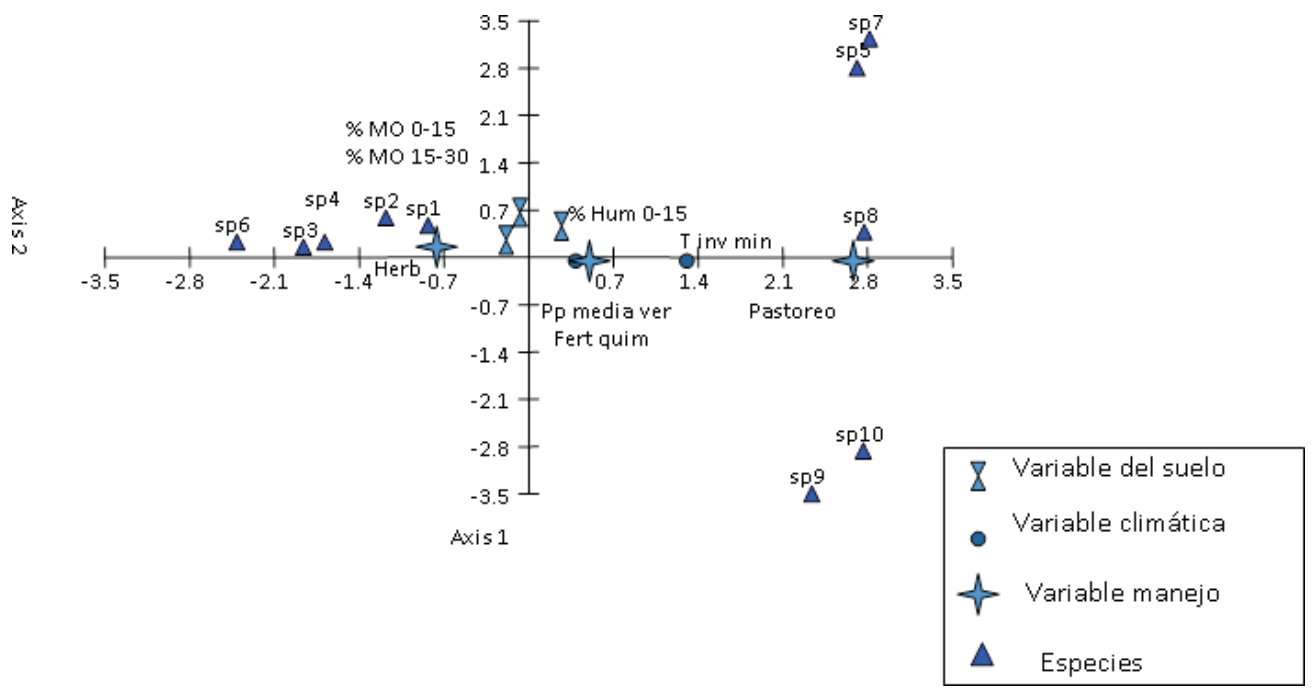

Figura 1. Tendencia de agrupación de especies de Melolonthidos según las variables que más influyen en su distribución en dos sistemas de producción tradicional de maíz. Región Purhépecha, Michoacán. México. (Scatter plot Análisis de Correspondencia con 18 variables y 8 casos). 
Pérez-Agis et al.: Abundancia de larvas de Melolonthidae en Michoacán, México

MATRIZ 1. Análisis de Correspondencia sin Tendencia (eigenvalue = puntuación de las variables). Se analizan 18 variables $* 8$ casos. Tolerancia eigenanalisis at $1 \mathrm{E}-7$

Eigenvalue Axis 1 Axis 2 Axis 3 Axis 4 Axis 5 Axis 6 Axis 7 Axis 8 Axis 9

$\begin{array}{llllllllll}\text { Eigenvalue } & 0.137 & 0.013 & 0.002 & 0.001 & 0.001 & 0 & 0 & 0 & 0\end{array}$

$\begin{array}{llllllllll}\text { Porcentaje } & \mathbf{6 2 . 4 4} & \mathbf{5 . 9 7} & \mathbf{1 . 0 3} & 0.515 & 0.325 & 0.171 & 0.056 & 0.051 & 0.051 \\ \text { Porcentaje } & \mathbf{6 2 . 4 4} & \mathbf{6 8 . 4} & \mathbf{6 9 . 4} & 69.957 & 70.28 & 70.453 & 70.51 & 70.561 & 70.612 \\ \text { Acumulado } & & & & & & & & & \end{array}$

Continua..

Eigenvalores Axis Axis Axis Axis Axis Axis Axis Axis Axis

$\begin{array}{lllllllll}10 & 11 & 12 & 13 & 14 & 15 & 16 & 17 & 18\end{array}$

$\begin{array}{llllllllll}\text { Eigenvalores } & 0 & 0 & 0 & 0 & 0 & 0 & 0 & 0 & 0\end{array}$

$\begin{array}{llllllllll}\text { Porcentaje } & 0.051 & 0.051 & 0.051 & 0.051 & 0.051 & 0.051 & 0.051 & 0.051 & 0.051\end{array}$

$\begin{array}{llllllllll}\text { Porcentaje } & 70.663 & 70.714 & 70.765 & 70.815 & 70.866 & 70.917 & 70.968 & 71.018 & 71.069\end{array}$

Acumulado

Puntuación de las variables

Axis 1 Axis 2 Axis 3 Axis 4 Axis 5 Axis 6 Axis 7 Axis 8 Axis 9

\begin{tabular}{ll|l|l|l|lllll} 
Hum 0-15 & 0.294 & $\mathbf{0 . 4 1}$ & $\mathbf{0 . 3 4}$ & 0.273 & 0.368 & 0.429 & 0.478 & 0.515 & 0.515
\end{tabular}

$\begin{array}{lllllllllll}\text { Materia Org. } & -0.105 & \mathbf{0 . 5 4} & \mathbf{0 . 6 4} & -0.455 & -0.565 & -0.65 & -0.548 & -0.554 & -0.552\end{array}$

0-15

$\begin{array}{llllllllll}\text { Materia Org. } & -0.058 & 0.181 & 0.162 & 0.223 & 0.253 & 0.266 & 0.238 & 0.242 & 0.241\end{array}$

15-30

$\begin{array}{lccccccccc}\text { Tem inv min } & \mathbf{1 . 2 6 7} & 0.035 & 0.034 & 0.302 & 0.287 & 0.228 & 0.183 & 0.18 & 0.179 \\ \text { Pp media ver } & 0.546 & 0.063 & 0.066 & 0.263 & 0.253 & 0.209 & 0.176 & 0.172 & 0.172 \\ \text { Fert quim } & 0.56 & 0.038 & 0.029 & 0.316 & 0.311 & 0.268 & 0.223 & 0.224 & 0.223 \\ \text { Herbicida } & -\mathbf{0 . 7 6} & 0.211 & 0.229 & 0.085 & 0.1 & 0.138 & 0.139 & 0.132 & 0.133 \\ \text { Descanso } & \mathbf{2 . 6 0 4} & 0.002 & -0.002 & 0.343 & 0.327 & 0.257 & 0.197 & 0.193 & 0.192 \\ \text { (pastoreo) } & & & & & & & & & \\ \text { sp1 } & -0.819 & 0.463 & 0.463 & 0.085 & 0.1 & 0.138 & 0.139 & 0.132 & 0.133 \\ \text { sp2 } & -1.163 & 0.572 & 0.712 & -0.586 & -0.77 & -0.983 & -0.853 & -0.855 & -0.852 \\ \text { sp3 } & -1.837 & 0.149 & 0.177 & 0.085 & 0.1 & 0.138 & 0.139 & 0.132 & 0.133 \\ \text { sp4 } & -1.663 & 0.222 & 0.238 & 0.085 & 0.1 & 0.138 & 0.139 & 0.132 & 0.133 \\ \text { sp5 } & 2.679 & 2.764 & 2.992 & -2.851 & -2.838 & -0.917 & 0.394 & 0.606 & 0.617 \\ \text { sp6 } & -2.378 & 0.222 & 0.238 & 0.085 & 0.1 & 0.138 & 0.139 & 0.132 & 0.133 \\ \text { sp7 } & 2.781 & 3.192 & 2.989 & -1.924 & -0.916 & 1.519 & 2.966 & 3.525 & 3.545 \\ \text { sp8 } & 2.737 & 0.369 & 1.424 & -3.155 & -5.266 & -9.449 & -9 & -8.96 & -8.948 \\ \text { sp9 } & 2.31 & -3.456 & -3.623 & 4.297 & 4.28 & 3.814 & 3.475 & 3.41 & 3.391 \\ \text { sp10 } & 2.73 & -2.828 & -2.41 & 2.131 & 1.472 & -2.54 & -5.022 & -5.57 & -5.594\end{array}$


Matriz 1. Continúa.

Continua...

\begin{tabular}{|c|c|c|c|c|c|c|c|c|c|}
\hline & $\begin{array}{c}\text { Axis } \\
10\end{array}$ & $\begin{array}{c}\text { Axis } \\
11\end{array}$ & $\begin{array}{c}\text { Axis } \\
12\end{array}$ & $\begin{array}{c}\text { Axis } \\
13\end{array}$ & $\begin{array}{c}\text { Axis } \\
14\end{array}$ & $\begin{array}{c}\text { Axis } \\
15\end{array}$ & $\begin{array}{c}\text { Axis } \\
16\end{array}$ & $\begin{array}{c}\text { Axis } \\
17\end{array}$ & $\begin{array}{c}\text { Axis } \\
18\end{array}$ \\
\hline Hum 0-15 & 0.515 & 0.515 & 0.517 & 0.517 & 0.517 & 0.517 & 0.517 & 0.517 & 0.517 \\
\hline $\begin{array}{l}\text { Materia Org. } \\
0-15\end{array}$ & -0.551 & -0.551 & -0.551 & -0.551 & -0.551 & -0.551 & -0.552 & -0.552 & -0.552 \\
\hline $\begin{array}{l}\text { Materia Org. } \\
15-30\end{array}$ & 0.241 & 0.241 & 0.242 & 0.242 & 0.242 & 0.242 & 0.242 & 0.242 & 0.242 \\
\hline Tem inv min & 0.179 & 0.179 & 0.179 & 0.179 & 0.179 & 0.179 & 0.179 & 0.179 & 0.179 \\
\hline Pp media ver & 0.171 & 0.171 & 0.171 & 0.171 & 0.171 & 0.17 & 0.171 & 0.171 & 0.171 \\
\hline Fert quim & 0.223 & 0.223 & 0.224 & 0.224 & 0.224 & 0.224 & 0.224 & 0.224 & 0.224 \\
\hline Herb & 0.133 & 0.133 & 0.13 & 0.13 & 0.13 & 0.13 & 0.13 & 0.13 & 0.13 \\
\hline $\begin{array}{l}\text { Descanso } \\
\text { (postoreo) }\end{array}$ & 0.192 & 0.192 & 0.192 & 0.192 & 0.192 & 0.192 & 0.192 & 0.192 & 0.192 \\
\hline sp1 & 0.133 & 0.133 & 0.13 & 0.13 & 0.13 & 0.13 & 0.13 & 0.13 & 0.13 \\
\hline sp2 & -0.851 & -0.85 & -0.85 & -0.85 & -0.849 & -0.849 & -0.85 & -0.85 & -0.85 \\
\hline sp3 & 0.133 & 0.133 & 0.13 & 0.13 & 0.13 & 0.13 & 0.13 & 0.13 & 0.13 \\
\hline sp4 & 0.133 & 0.133 & 0.13 & 0.13 & 0.13 & 0.13 & 0.13 & 0.13 & 0.13 \\
\hline sp5 & 0.621 & 0.624 & 0.628 & 0.63 & 0.631 & 0.631 & 0.631 & 0.631 & 0.631 \\
\hline sp6 & 0.133 & 0.133 & 0.13 & 0.13 & 0.13 & 0.13 & 0.13 & 0.13 & 0.13 \\
\hline sp7 & 3.551 & 3.557 & 3.571 & 3.573 & 3.575 & 3.576 & 3.573 & 3.573 & 3.573 \\
\hline sp8 & -8.944 & -8.94 & -8.923 & -8.921 & -8.92 & -8.92 & -8.921 & -8.92 & -8.92 \\
\hline sp9 & 3.385 & 3.38 & 3.386 & 3.384 & 3.382 & 3.381 & 3.377 & 3.377 & 3.376 \\
\hline sp10 & -5.601 & -5.608 & -5.603 & -5.606 & -5.608 & -5.609 & -5.611 & -5.611 & -5.612 \\
\hline
\end{tabular}

Puntuación de los casos

$\begin{array}{lccccccccc} & \text { Axis 1 } & \text { Axis 2 } & \text { Axis 3 } & \text { Axis 4 } & \text { Axis 5 } & \text { Axis 6 } & \text { Axis 7 } & \text { Axis 8 } & \text { Axis 9 } \\ \mathbf{u 1} & 0.391 & 0.128 & 0.135 & 0.202 & 0.189 & 0.146 & 0.129 & 0.128 & 0.128 \\ \mathbf{u 2} & 0.241 & 0.176 & 0.196 & 0.121 & 0.093 & 0.044 & 0.046 & 0.046 & 0.046 \\ \mathbf{u 3} & 0.463 & 0.099 & 0.099 & 0.246 & 0.241 & 0.206 & 0.181 & 0.18 & 0.18 \\ \mathbf{u 4} & 0 & 0.186 & 0.212 & 0.092 & 0.059 & 0 & 0.003 & 0.002 & 0.002 \\ \mathbf{s f 1} & 0.593 & 0.2 & 0.212 & 0.119 & 0.11 & 0.138 & 0.175 & 0.185 & 0.185 \\ \mathbf{s f 2} & 0.671 & 0.162 & 0.193 & 0.107 & 0.067 & 0.001 & 0 & 0 & 0 \\ \mathbf{s f 3} & 0.598 & 0 & 0 & 0.349 & 0.341 & 0.282 & 0.245 & 0.242 & 0.241 \\ \mathbf{s f 4} & 0.711 & 0.313 & 0.331 & 0 & 0 & 0.102 & 0.182 & 0.202 & 0.203\end{array}$


Pérez-Agis et al.: Abundancia de larvas de Melolonthidae en Michoacán, México

Matriz 1. Continúa.

Continua...

$\begin{array}{lccccccccc} & \text { Axis } & \text { Axis } & \text { Axis } & \text { Axis } & \text { Axis } & \text { Axis } & \text { Axis } & \text { Axis } & \text { Axis } \\ \text { u1 } & 10 & 11 & 12 & 13 & 14 & 15 & 16 & 17 & 18 \\ \mathbf{u 2} & 0.128 & 0.128 & 0.128 & 0.128 & 0.128 & 0.128 & 0.128 & 0.128 & 0.128 \\ \mathbf{u 3} & 0.046 & 0.046 & 0.046 & 0.046 & 0.046 & 0.046 & 0.046 & 0.046 & 0.046 \\ \mathbf{u 4} & 0.18 & 0.18 & 0.179 & 0.179 & 0.179 & 0.179 & 0.179 & 0.179 & 0.179 \\ \mathbf{s f 1} & 0.002 & 0.002 & 0.002 & 0.002 & 0.002 & 0.002 & 0.002 & 0.002 & 0.002 \\ \mathbf{s f 2} & 0.185 & 0.186 & 0.185 & 0.185 & 0.185 & 0.186 & 0.186 & 0.186 & 0.186 \\ \text { sf3 } & 0 & 0 & 0 & 0 & 0 & 0 & 0 & 0 & 0 \\ \text { sf4 } & 0.24 & 0.24 & 0.24 & 0.24 & 0.24 & 0.24 & 0.24 & 0.24 & 0.24 \\ & 0.203 & 0.203 & 0.203 & 0.203 & 0.203 & 0.203 & 0.203 & 0.203 & 0.203\end{array}$

Felipe. Por otro lado, un tercer grupo estuvo formado por las especies sp. 6 (Paranomala sp. 2), sp. 3 (P. dentex), sp. 4 (Phyllophaga sp. 4), sp. 2 (Macrodactylus sp.) y sp. 1 (I. arizonensis) en orden decreciente, situadas en el mismo eje izquierdo con valores negativos, donde la aplicación de herbicida principalmente parece explicar su agrupación, posiblemente seguida por la influencia de la precipitación media de verano y la aplicación de fertilizante químico, todas estas especies estuvieron presentes en San Francisco Uricho, la localidad con manejo intensivo (Cuadro 2 y Fig. 1).

Agrupación de los sitios.- En la figura 2 se presenta la agrupación de los sitios, en donde claramente se muestran dos grupos. Hacia la izquierda las parcelas de la localidad de Uricho y hacia la derecha las de San Felipe de los Herreros, en ambos casos las variables de manejo hacen la diferencia ya que la mayoría de las variables ambientales se concentran en el centro en la Figura 1, lo que indica menor dispersión de las variables y por lo mismo menor influencia. Al analizar las dos figuras anteriores y la puntuación que arrojó el análisis (Matriz 1), se puede suponer que en Uricho las variables de aplicación de herbicida y fertilización química explican su agrupación, ya que según los resultados de la matriz de salida para este primer conjunto de especies la puntuación de herbicida (-0.757) explica la dispersión de las especies en el primer eje, mientras que para San Felipe es el periodo de descanso con libre pastoreo.

\section{DISCUSIÓN}

Los resultados obtenidos muestran como las variables relacionadas con las prácticas de manejo del sistema de producción, como periodos de descanso con libre pastoreo y aplicación de herbicida influyen en la distribución de la especies. Se corrobora que la presencia del complejo gallina ciega (CGC) está muy relacionado con la disponibilidad de materia orgánica y humedad en el suelo. También se muestra como las 


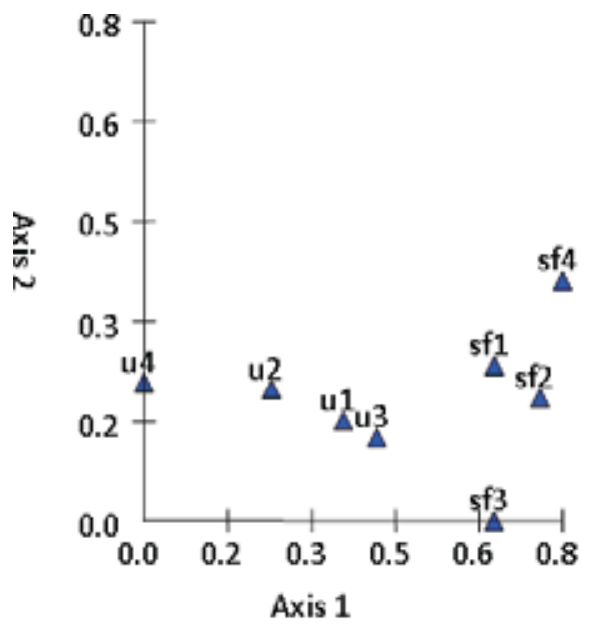

Sitios de muestreo (Parcelas)

Figura 2. Tendencia de distribución de los sitios de muestreo por sistemas de manejo para la producción de maíz. Región Purhépecha, Michoacán. México. (Scatter plot Análisis de Correspondencia con 25 variables y 8 casos).

variables de suelo influyen menos que las del clima.

En San Francisco Uricho la especie que más se asocia al periodo de crecimiento de las plantas de maíz es Phyllophaga sp. 4 (sp. 4) y este análisis sugiere que entre las variables de manejo que pueden influir más en su abundancia es la aplicación de herbicida y el porcentaje de materia orgánica en los primeros $15 \mathrm{~cm}$ de suelo. Aparentemente esto también aplica para Paranomala sp. 2 (sp. 6), y P. dentex (sp. 3).

En San Felipe de los Herreros el descanso con libre pastoreo y la temperatura de invierno mínima promedio explican la abundancia de $P$. vetula (sp. 8) Pa. punctatipennis (sp. 7), y P. platyrhina (sp. 5). Mientras que para Macrodactylus sp. (sp. 2) e I. arizonensis (sp 1), la puntuación de las variables del suelo, porcentaje de humedad de 0 a $15 \mathrm{~cm}$ (con 0.405$)$ y porcentaje de materia orgánica a la misma profundidad (con 0.538) explican la dispersión de las especies en el segundo eje.

\section{CONCLUSIONES}

Los melolontidos de importancia agrícola frecuentemente se han estudiado como una plaga de los sistemas de producción, principalmente por los hábitos fitófagos de algunas especies, sin embargo en el CGC hay otras especies que aún se desconoce sus hábitos alimenticios y que muy probablemente juegan un papel importante en la ecología del suelo y concretamente de los agroecosistemas maiceros de México. 
Este documento es un acercamiento para tratar de identificar algunas variables que probablemente influyen en la presencia de las especies del CGC en dos sitios específicos, a partir de este ejercicio es posible mejorar la selección de variables para tratar de entender que puede influir en la composición del CGC y su riqueza biológica en sitios determinados.

Los resultados de este documento sugieren que las prácticas de manejo de la fertilidad del agroecosistema son importantes en la distribución de las especies del CGC.

Finalmente sí a través de prácticas de manejo se lograra minimizar la dominancia de las especies fitófagas y aumentar o diversificar con especies consumidoras de detritus el CGC, se esperaría que en el mediano y largo plazo las poblaciones se autoregularan (Altieri \& Nicholls 1999) y probablemente el CGC resultara ser un aliado en el manejo de los agroecosistemas maiceros y temporaleros del país.

AGRADECIMIENTOS. La participación de las autoridades agrarias y los productores de las localidad nativas de la cultura Purhépecha San Felipe de los Herreros y San Francisco Uricho fue fundamental para la realización del presente estudio. Así mismo, la orientación bioestadística del Dr. Javier Ponce, las facilidades del laboratorio otorgadas por el Dr. Juan Manuel Sánchez Yáñez de la UMSH, el Técnico Valdemar Morales Soto de PAIR Occidente A.C., y las recomendaciones del Dr. Sergio Zamudio del INECOL (Pátzcuaro) fueron pilar de este documento.

\section{LITERATURA CITADA}

Altieri, M. \& Nicholls, C. 1999. Biodiversity, ecosystem function, and insect pest management in agricultural systems, pp. 120-132. In: Collins W. \& C. Qualset. (Eds.) Biodiversity in agroecosystems. CRC Press. USA.

Braak, ter, C. J. F. 1995. Ordination, pp. 91-173. In: Jongman, R. H. G., C. J. K. ter Braak \& O. F. R. Van Tongeren (Eds.). Data Analysis in Community and Landscape Ecology. Cambridge University Press, United Kingdom.

Brown, G., Fragoso C., Barois, I., Rojas, P., Patrón, J.C., Bueno, J., Moreno, A.G., Lavalle, P. \& Ordáz, V. 2001. Diversidad y rol funcional de la macrofauna edáfica en los ecosistemas tropicales, pp. 79-110. In: Fragoso, C. \& P. Reyes-Castillo (Eds.). Diversidad, función y manejo de la biota edáfica en México. Acta Zoológica Mexicana (n.s.) Número especial 1.

Büchs, W. 2003. Biodiversity and agri-environmental indicators - general scopes and skills with special reference to the habitat level, pp. 35-78. In: Wolfgan Büchs (Ed.). Biotic indicators for biodiversity and sustainable agriculture. Agriculture Ecosystems and Environment. Special Issue. Elsevier 98.

Fragoso, C., Reyes-Castillo, P. \& Rojas, P. 2001. La importancia de la biota edáfica en México, pp. 1-10. In: C. Fragoso \& P. Reyes Castillo (Eds.) Diversidad, función y manejo de la biota edáfica en México. Acta Zoológica Mexicana (n. s.) Número especial 1.

Morón, M. A. 1997. White grubs (Coleoptera: Melolonthidae: Phyllophaga Harris) in México and Central America. A brief review. Trends in Entomology, 1: 117-128.

Morón, M. A. 2010. Diversidad y distribución del complejo "gallina ciega" (Coleoptera: Scarabaeoidea), pp. 41-63. In: Rodríguez del Bosque, L. A. \& M. A. Morón (Eds.). Plagas del suelo. MundiPrensa, México.

Pérez-Agis, E., Morón, M. A., Nájera-Rincón, M. B., López-Barbosa, E. \& Vázquez-García, M. 2008. Análisis de diversidad del "complejo gallina ciega" (Coleoptera: Melolonthidae) en dos sis- 
temas de producción tradicional de maíz en la Región Purhépecha, Michoacán. Acta Zoológica Mexicana (n.s.), 24: 221-235.

Ramírez-Salinas, C. \& Castro-Ramírez, A. E. 2000. El complejo "gallina ciega" (Coleoptera: Melolonthidae) en el cultivo de maíz en El Madronal, municipio de Amatenango del Valle, Chiapas, México. Acta Zoológica Mexicana (n.s.), 79: 17-41.

Romero-López, A. A., Morón M. A., Aragón A. \& Villalobos, F. J. 2010. La "gallina ciega" (Coleoptera: Scarabaeoidea: Melolonthidae) vista como un "ingeniero del suelo". Southwestern Entomologist, 35: 331-343. 